\title{
Inteligencia Emocional en jóvenes Voluntarios y No voluntarios del Gran Concepción*
}

\author{
Claudia Quiroga Sanzana** \\ Isis Chamblás García***
}

RESUMEN ${ }^{1}$

\begin{abstract}
Este artículo hace referencia al nivel de inteligencia emocional que presentan jóvenes Universitarios de Concepción, cuya diferencia principal es la realización de voluntariado. La investigación es de carácter cuantitativo y corte transversal. Para medir Inteligencia Emocional se utilizó el test BarOn ICE. Se trabajó con un grupo perteneciente a una agrupación formal de voluntariado y jóvenes que no realizan estas acciones, siendo similares en otras características al grupo anterior. Estos jóvenes fueron convocados a través de las respectivas Jefaturas de Carrera. Tras el análisis, se observa que en la mayoría de los componentes de la inteligencia emocional, son los voluntarios quienes presentan mayores puntajes, diferencias que resultan significativas sólo en las subescalas "Solución de Problemas" y "Control de impulsos".
\end{abstract}

Palabras clave: Inteligencia Emocional, Voluntarios, No voluntarios, BarOn ICE.

\section{Inteligência emocional em jovens Voluntários e Não voluntários da grande Conceição}

RESUMO

Este artigo se refere ao nível de inteligência emocional que apresentam os jovens Universitários de Conceição, cuja diferença principal é a realização do voluntariado. A pesquisa é de caráter quantitativo e de corte transversal. Para medir a inteligência emocional utilizou-se o teste Barão ICE. Trabalhou-se com um grupo pertencente a um agrupamento formal de voluntariado e jovens que não realizam estas ações, sendo semelhantes em outras características ao grupo anterior. Estes jovens foram convidados

Artículo recibido: 25/05/2014. Artículo aprobado: 03/09/2014. Versión Final: 08/10/2014.

** Chilena. Trabajadora Social, Magíster en Trabajo Social y Políticas Sociales, Universidad de Concepción, Chile. Trabajadora Social en Centro para el Desarrollo de Microempresas (CDM). Docente en Instituto Profesional AIEP. Correo electrónico: claudiaquiroga@udec.cl

*** Chilena. Trabajadora Social, Magíster en Educación para el Trabajo Social, Universidad Católica de América, Washington D.C. Profesora Asociada Departamento de Trabajo Social, Facultad de Ciencias Sociales, Universidad de Concepción, Chile. Correo Electrónico: ichambla@udec.cl

1 Este artículo se deriva de la Tesis de Claudia Quiroga para optar al Grado de Magíster en Trabajo Social y Políticas Sociales, titulada "Acciones de voluntariado y su impacto en el desarrollo de competencias en el voluntario". Universidad de Concepción, Chile. 
através das respectivas Chefaturas de Carreira. Após a análise, observase que na maioria dos componentes da inteligência emocional, são os voluntários que apresentam maiores pontuações, diferenças que são significativas só nas subescalas.

Palavras-chave: Inteligência Emocional, Voluntários, Não voluntários, Barão ICE.

\section{Emotional Intelligence in Young Volunteers and Non Volunteers in Concepcion}

ABSTRACT

This article refers to the emotional intelligence level of young students of Universidad de Concepcion, whose main difference is volunteering. The research is quantitative and cross-sectional. It has used the BarOn ICE test to measure emotional intelligence in a group formally working as volunteers and another group of students who do not but having other similar characteristics. These young people were invited by the respective Career Head. After the analysis, it is observed that most of the components of emotional intelligence are present in the volunteer group and differences are significant only in the subscales "Troubleshooting" and "Impulse control."

Keywords: Emotional intelligence, volunteers, non-volunteers, BarOn ICE.

\section{Antecedentes}

El voluntariado es conocido como aquella actividad que se realiza en beneficio de otro, de manera libre, espontánea, en forma sistemática y sin recibir remuneración a cambio (Alcalá Consultores, 2002). Surge y se desarrolla con la humanidad como una forma de dar respuesta a injusticias sociales y como una forma de apoyo para quienes se encuentran en una situación de carencia, o viven una injusticia (Delgadillo, 2005). A lo largo de la historia, ha sido posible constatar la evolución de estas acciones ya sea desde la cultura oriental como también la occidental: De acuerdo a Román (2011), su surgimiento ha sido como parte de acciones de caridad emprendidas por la Iglesia Católica; incluso en el Chile actual, aun se percibe a ésta misma institución como la que encauza mayormente las acciones de voluntariado (Román, 2011).

En la literatura consultada (Alcalá Consultores, 2002; Delgadillo, 2005; Rodríguez, 2000; Zulueta, 2003) el voluntariado ha sido mayoritariamente estudiado desde la perspectiva del aporte hacia quien se apoya, pero no existe una línea clara de análisis desde la 
figura del sujeto voluntario. Según la Organización de las Naciones Unidas (ONU) (2000), el voluntariado a nivel mundial tiene dos grandes beneficios para las naciones y en ellos radica la importancia de fomentar su desarrollo por parte de los Estados; por un lado, un beneficio de orden económico, ya que las actividades realizadas por voluntarios, liberan lo que podría ser financiado por el Estado o por privados, y, por otro lado, un segundo beneficio se relaciona con el fomento de la confianza entre los ciudadanos y la contribución que tiene el voluntariado en la elaboración de normas de solidaridad y reciprocidad en cada cultura. Asimismo, Rodríguez (2000) hace mención a la importancia del voluntariado, relacionándolo con el hecho de que este fenómeno se orienta a fomentar el desarrollo de la responsabilidad de las personas, en relación a lo que ocurre a su alrededor, sobre todo en los jóvenes y en el contexto de las universidades. En la misma línea, Zulueta (2003) agrega que el voluntariado no es sólo una buena opción y una ayuda para trabajar en pro de la superación de la pobreza, sino que también es una forma de potenciar la solidaridad en las personas que no sufren esa exclusión. Rodríguez (2000) y Zulueta (2003) plantean en general, que los aportes del voluntariado no tan sólo surten efecto en las personas que reciben la ayuda, sino además en la sociedad en su conjunto, puesto que su ejecución fomenta el desarrollo de diversos valores, como la solidaridad, la empatía, la capacidad participativa, la buena disposición, entre otros. Quizás el efecto más observable sea el que el voluntario realiza en sí mismo, en su vida, en sus relaciones, en el propio compromiso profesional, en la medida que transfiera coherentemente a la actividad que realiza de forma cotidiana, aquellos valores que experimenta (o refuerza) en la acción del voluntariado. Si se lograse, por tanto, esta conexión de los valores del voluntariado y la vida diaria, podría plantearse que el voluntariado tiene efectos en la sociedad.

La presente investigación se enmarca en la perspectiva descrita; el propósito central fue estudiar el aporte del voluntariado, más allá del beneficio de quien lo recibe, sino más bien centrado en el que ejecuta esta acción -el voluntario-, y así observar en qué medida la participación en estas acciones impactan favorablemente en el desarrollo de "capacidades distintas", como mayor empatía, liderazgo, o mejores relaciones con otros, las cuales en conjunto representan la Inteligencia Emocional, entendida -en esta investigación-como el "conjunto de habilidades personales, emocionales y sociales y de destrezas que influyen en nuestra habilidad 
para adaptarnos y enfrentar las demandas y presiones del medio" (Baron, 1997, en Ugarriza, 2001: 131).

Los estudios de Inteligencia Emocional se inician con Gardner en 1983, quien afirma que la inteligencia comprende múltiples dimensiones, combinando una variedad de aspectos cognoscitivos con elementos de la inteligencia personal (como él la llamó inicialmente); esta dimensión comprendía dos componentes generales que él refirió como "capacidades intrapersonales" y "habilidades interpersonales". Ya en 1990, Salovey y Mayer, fueron los primeros en utilizar el término "Inteligencia Emocional", refiriéndose a ella como "una forma de inteligencia social que implica la habilidad para dirigir los propios sentimientos y emociones, así como de los demás, saber discriminar entre ellos y usar esta información para guiar el pensamiento y la propia acción" (Salovey y Mayer, 1990: 186). Goleman interpreta y resume las dos inteligencias de Gardner, llegando a lo que es la inteligencia emocional, refiriendo que es un "Conjunto de destrezas, actitudes, habilidades y competencias que podrían determinar la conducta del individuo, sus reacciones, estados mentales, y que puede definirse como la capacidad para reconocer los propios sentimientos y de los demás, de motivarse y de manejar adecuadamente las relaciones" (1995: 29). Con ello se explicaría por qué ciertas personas con una elevada inteligencia emocional pueden tener mayores logros a lo largo de su vida, que aquellas que tienen un mayor coeficiente intelectual. Esta inteligencia no viene dada por sí misma al nacer, sino que se puede crear y fortalecer a través de una combinación de factores, entre ellos, las experiencias desde la infancia y durante el desarrollo de la vida (Goleman, 1995). Por tanto, el impacto que tiene la sociedad en el desarrollo de la inteligencia emocional de los sujetos en muy amplio, puesto que el ser humano está destinado a vivir rodeado de otros.

Por otro lado, contar con la capacidad de poder relacionarse de buena forma y de comprenderse a uno mismo, resulta una ventaja para la sociedad en su conjunto, en la medida que cuenta con individuos que pueden insertarse de mejor manera, que pueden tener mejor calidad de relaciones y que por tanto van a significar mayor aporte (Goleman, 2006). De esta manera, la inteligencia no-cognitiva (emocional) es un factor importante en la determinación de la habilidad para tener éxito en la vida, influyendo directamente en el bienestar general. En ese sentido, el hecho de 
centrar la mirada en los propios protagonistas de la acción voluntaria, apunta a rescatar los elementos más subjetivos sin dejar de lado los aspectos que influencian y son influidos por las subjetividades particulares. Esto como una forma de demostrar que estudiar este fenómeno puede provocar un beneficio mayor a nivel social, puesto que se generan con ello nuevos conocimientos en el ámbito de las competencias sociales y, a su vez, contribuye a la reflexión acerca de la importancia de éstas en la vida cotidiana y particularmente en la formación académica, espacio que resulta propicio para potenciar dichas competencias, prioritarias en la formación de un profesional integral, sea o no del área social, particularmente cuando se busca un mejor desarrollo de nuestra sociedad, el que se logra, también, con el desarrollo de las personas que la componen.

\section{Material y método}

La investigación realizada corresponde a un tipo de estudio cuantitativo, cuasi-experimental y de corte transversal. La investigación toma como base el Modelo Rubin, que extrapola las características del experimento verdadero a este tipo de estudio, en la medida que se requiere contar con un grupo tratamiento y un grupo control, para establecer si efectivamente la variable tratamiento (participación en voluntariado) es lo que estaría generando mayores diferencias. El estudio básicamente buscó establecer si el realizar actividades voluntarias tiene incidencia sobre la Inteligencia emocional de los jóvenes y los componentes de ésta (interpersonales, intrapersonales, de adaptabilidad, manejo del estrés y estado de ánimo).

La población de estudio correspondió a jóvenes universitarios, por una parte el grupo experimental o tratamiento, lo conformaron jóvenes que desarrollaban actividades de voluntariado organizadas por el grupo "Voluntarios por la Educación y Cultura" $(\mathrm{VEC})^{2}$. El número de integrantes fue de 40 jóvenes, por lo que se trabajó con ese universo.

Por otra parte, el grupo control estuvo conformado por aquellos estudiantes de la Universidad de Concepción que presentaron ca-

Grupo que nace al alero de la Universidad de Concepción, con la idea de aportar con su tiempo en lugares de la Región del Biobío que se encuentren con algún tipo de carencia. 
racterísticas similares con el grupo experimental en variables como sexo, edad y carrera de estudio, y que no realizaban voluntariado.

La propuesta inicial era acceder al mayor número de casos posible, de tal manera de poder seleccionar los sujetos de mayor similitud con el grupo tratamiento a través del sistema de "pareo de casos". Finalmente se optó por trabajar con la totalidad de los sujetos que respondieron al cabo de 3 meses y que fueron un total de 262. Se utilizó el método de encuesta, por medio de la aplicación del cuestionario autoadministrado.

\section{Instrumento de encuesta}

Para la medición de inteligencia emocional, se utilizó el Test/Inventario de Inteligencia Emocional de Bar-On (Bar-On I-CE). Este instrumento fue elaborado por Reuven Bar-On con la denominación original de EQ-I (Emotional Quotient Inventory), traducido y revisado en el Perú por Zoila Abanto, Leonardo Higueras y Jorge Cueto, en el año 2000 (Sotil y Quintana, 2002). Este test puede ser aplicado de forma individual y colectiva, a personas mayores de 16 años. Permite evaluar 5 componentes de la Inteligencia emocional, subdivididos en 15 subescalas: Componente Intrapersonal: Comprensión de sí mismo, autoconcepto, asertividad, independencia y autorrealización. Componente Interpersonal: Empatía, relaciones interpersonales y responsabilidad social; Componente de Adaptabilidad: Solución de problemas, prueba de la realidad, y flexibilidad; Componente del Manejo de Estrés: Tolerancia al estrés y control de impulsos; Componente del estado de Ánimo en General: Felicidad y optimismo. Posee 133 ítems, 64 de ellos están planteados de forma negativa y 69 , de manera positiva. Cada uno de ellos tiene cinco alternativas de respuesta.

Los resultados que se pueden obtener son: Un resultado de Cuociente emocional total, de los 5 componentes y de las 15 subescalas antes mencionadas (Abanto, Higueras y Cueto, 2000, en Sotil y Quintana, 2002). El índice interpretativo de la inteligencia emocional cuenta con 7 niveles: Marcadamente alta, Muy alta, alta, Promedio, baja, muy baja y marcadamente baja. La confiabilidad se obtuvo por el método de consistencia interna con Alfa de Cronbach, obteniendo coeficientes para las quince subescalas que miden entre 0.69 y 0.86 , con un coeficiente de consistencia interna total de 0.76 (Sotil y Quintana, 2002). 
En Perú no se ha medido la confiabilidad por retest (Ugarriza, 2001), pero sí lo hizo Bar-On en 1997, obteniendo un coeficiente de confiabilidad promedio para las quince subescalas de 0.85 después de un mes y de 0.75 después de cuatro meses (Ugarriza, 2001). En relación a la validez, ésta ha sido analizada por 9 estudios de validación: Validez de contenido, aparente, de constructo, factorial, convergente, divergente, de criterio de grupo, discriminante y predictiva; siendo posible encontrar en todos los casos "coeficientes de validez claramente adecuados" (Sotil y Quintana, 2002).

Para la medición de características sociodemográficas y familiares se elaboró una pauta de preguntas que abordaban estas variables.

\section{Trabajo de Campo}

- Acceso al Grupo Tratamiento: Para llevar a cabo la investigación, fue necesario solicitar la colaboración del grupo de voluntarios VEC, que se encuentra en la Universidad de Concepción. Para ello, se tomó contacto con sus coordinadores, con el objetivo de llevar a cabo una reunión y solicitar su ayuda en el contacto con los voluntarios y la posibilidad de aplicar el instrumento en una de sus reuniones y contactar vía correo electrónico a quienes no se encontrasen presentes en ese momento. En dicha reunión, los coordinadores sugirieron crear un link que pudiese ser enviado a sus correos electrónicos, ya que en las reuniones el tiempo era escaso y la tasa de asistencia era muy variable. Tomando en cuenta la propuesta de los coordinadores, el procedimiento utilizado fue el siguiente: La tesista acudió a una de las reuniones del grupo, solicitó la colaboración de los jóvenes y sus correos electrónicos.

El instrumento fue enviado directamente a cada joven voluntario a través de un enlace electrónico, donde solo debía ingresar y contestar. De manera automática, las respuestas eran derivadas a una planilla de respuestas, asignándole un código a cada uno. Toda vez que se obtuvo la respuesta de los 40 voluntarios, se revisó en detalle las características del grupo, es decir, de qué carreras provenían, grupo de edad y sexo.

- Acceso al Grupo Control: Una vez tomado el conocimiento de estas características, fue posible decidir a quiénes contactar. Para ello se tomó contacto con cada uno de los Jefes de Carrera, a los cuales pertenecían los voluntarios. El primer contacto fue vía correo electrónico, y posteriormente se sostuvo una reunión con la 
mayoría de ellos, donde se entregó una carta explicando lo que se requería. En dicha carta, entre otras cosas, se exponía a quién era necesario contactar de su carrera, se daba la posibilidad de escoger el envío del enlace electrónico con el cuestionario a ciertos alumnos, o bien para facilitar la labor del Jefe de Carrera, enviarlo a todos los estudiantes de la carrera, solicitando responder a quienes reunían ciertas características señaladas de antemano, indicando además el carácter voluntario. En total, 262 jóvenes dieron respuesta al cuestionario.

A cada participante, tanto del grupo de voluntarios como los no voluntarios, se solicitó su participación a través de un consentimiento informado, indicando el carácter confidencial de la información y dejando libertad para participar de la misma.

- Análisis de datos. El análisis fue realizado utilizando el programa estadístico Statistical Analysis System (SAS).

\section{Resultados}

\section{Características sociodemográficas}

De un total de 302 estudiantes voluntarios y no voluntarios, un $61,3 \%$ corresponde a mujeres; tendencia que se presenta en ambos grupos (sobre 60\%). La edad de la muestra total, fluctúa entre los 18 y los 29 años. El grupo etario donde se concentra mayor proporción de estudiantes voluntarios tiene entre 1821 años (50\%) respecto de los no voluntarios (33,6\%), quienes mayoritariamente $(62,6 \%)$ tienen entre $22-25$ años. La mayoría de los estudiantes provienen de las comunas que componen el gran Concepción (Concepción, Talcahuano, Hualpén, San Pedro de la Paz, Chiguayante, Hualqui, Penco, Tomé, Coronel, y Lota), superando el $97 \%$ en cada grupo estudiado; respecto de la religión, la mayoría de la muestra se identifica con alguna religión $(64,9 \%)$, siendo mayor el porcentaje de los voluntarios $(85 \%)$ respecto de los no voluntarios $(61,8 \%)$. De acuerdo a la situación civil, un $97 \%$ de la muestra total declara estar soltero; el $5 \%$ de los voluntarios y el $2,7 \%$ de los no voluntarios se declara casado o conviviendo.

Las áreas de estudios que predominan en los voluntarios corresponden a carreras de las Ciencias Sociales (30\%), Biología (22,5\%) e ingeniería (20\%), tendencia que se mantiene en los no volunta- 
rios. La mayoría de los estudiantes indica que ningún miembro de su familia realiza actividades de voluntariado $(89,4 \%)$, siendo mayor el porcentaje en los no voluntarios $(92,4 \%)$ respecto de los voluntarios (70\%). Con relación al parentesco que tienen con aquella persona que realiza actividades de voluntariado, se observa que, primeramente, son los hermanos quienes "modelan" en esta experiencia, porcentaje que es mayoritario en el grupo de voluntarios $(27,5 \%)$ seguido por los primos $(2,5 \%)$. En los no voluntarios sólo el 7,6\% tiene familiares con experiencias de voluntariado, de los cuales el 3,4\% corresponde a hermanos y el 2,7\% a mamá o papá; esta última situación no está presente en los voluntarios.

\section{Inteligencia Emocional en Voluntarios y No Voluntarios}

Respecto al Nivel de inteligencia emocional y de acuerdo a las categorías establecidas por Baron (2000), los no voluntarios concentran un porcentaje cercano al 50\% en un nivel "promedio", mientras que los voluntarios se distribuyen entre la categoría "alta" y "promedio" cerca de un 70\%. Comparativamente, el 52,5\% de estos últimos, respecto del 26\% de los no voluntarios, obtienen puntajes que los ubican en las categorías de "alta" a "marcadamente alta". Cabe destacar que en los voluntarios no se encuentran estudiantes en la categoría "marcadamente baja" y un solo caso en "muy baja", versus el 13,4\% observado en su contraparte, en estas dos categorías indicadas (Tabla $\mathrm{N}^{\circ} 1$ ).

Con relación a los promedios de los puntajes directos y ponderados, el nivel global de Inteligencia Emocional en ambos grupos de estudio y de acuerdo a la categorización previamente indicada, se puede calificar de Capacidad desarrollada de forma "adecuada" (promedio).

Aun cuando ambos grupos obtienen una categorización global igual, los puntajes promedios reales y ponderados, muestran que los voluntarios superan a los no voluntarios, tendencia que se reconoce también a nivel de cada uno de los componentes, diferencias que aparecen significativas al aplicar la prueba t de Student en los 5 componentes por separado, y en el promedio de Inteligencia emocional total $\left(\mathrm{t}=-2.97\right.$; $\mathrm{p}<0.0032$ ) (Tabla $\mathrm{N}^{\circ} 2$ ) 
Tabla 1. "Nivel de Inteligencia emocional global, según condición de voluntario"

\begin{tabular}{|l|l|l|l|l|}
\hline \multirow{2}{*}{ Inteligencia Emocional } & \multicolumn{3}{|l|}{ Voluntarios $(\mathrm{N}=40)$} & \multicolumn{2}{l|}{ No voluntarios $(\mathrm{N}=262)$} \\
\cline { 2 - 6 } & $\mathrm{Fi}$ & $\%$ & $\mathrm{Fi}$ & $\%$ \\
\hline Marcadamente Alta & 1 & 2.5 & 4 & 1.5 \\
\hline Muy alta & 6 & 15 & 23 & 8.8 \\
\hline Alta & 14 & 35 & 41 & 15.7 \\
\hline Promedio & 14 & 35 & 130 & 49.6 \\
\hline Baja & 4 & 10 & 29 & 11 \\
\hline Muy baja & 1 & 2.5 & 18 & 6.9 \\
\hline Marcadamente baja & - & - & 17 & 6.5 \\
\hline Total & 40 & 100 & 262 & 100 \\
\hline
\end{tabular}

Tabla 2. "Promedios Inteligencia Emocional Global y por Componentes, según condición de Voluntario"

\begin{tabular}{|c|c|c|c|c|c|c|c|c|}
\hline \multirow{2}{*}{$\begin{array}{l}\text { Componentes } \\
\text { Inteligencia } \\
\text { Emocional }\end{array}$} & \multicolumn{3}{|c|}{$\begin{array}{l}\text { Voluntarios } \\
(\mathrm{N}=40)\end{array}$} & \multicolumn{3}{|c|}{$\begin{array}{l}\text { No voluntarios } \\
(\mathrm{N}=262)\end{array}$} & \multicolumn{2}{|c|}{$\begin{array}{l}\text { Prueba T de } \\
\text { Student }\end{array}$} \\
\hline & & $\mathrm{Pp}$ & Ds & & $\mathrm{Pp}$ & Ds & $\mathrm{t}$ & $\mathrm{p}$ \\
\hline Intrapersonal & 158 & 100 & 18.25 & 150 & 93 & 22.32 & -2.02 & 0.0446 \\
\hline Interpersonal & 104 & 108 & 8.46 & 98 & 99 & 10.72 & -3.63 & 0.0006 \\
\hline Adaptabilidad & 99 & 104 & 11.01 & 94 & 97 & 12.40 & -2.11 & 0.0358 \\
\hline $\begin{array}{l}\text { Manejo del } \\
\text { Estrés }\end{array}$ & 73 & 111 & 10.00 & 68 & 104 & 10.21 & -2.93 & 0.0037 \\
\hline $\begin{array}{l}\text { Estado del } \\
\text { ánimo en } \\
\text { general }\end{array}$ & 72 & 106 & 8.36 & 68 & 99 & 10.59 & -3.07 & 0.0032 \\
\hline $\begin{array}{l}\text { Total } \\
\text { Inteligencia } \\
\text { Emocional }\end{array}$ & 475 & 108 & 51.01 & 450 & 99 & 42.67 & -2.97 & 0.0032 \\
\hline
\end{tabular}

\section{Inteligencia Emocional por Componentes y Subescalas en Voluntarios y No Voluntarios}

Al analizar el Componente Intrapersonal, a través del comportamiento de las subescalas que la componen, en la mayoría de ellas, los puntajes promedios observados, tanto real como ponderado, son mayores en los voluntarios, superando a los no voluntarios entre 4 a 7 puntos, excepto en "Asertividad" e "Independencia", donde los puntajes son iguales en ambos grupos. 
Por otra parte, y aun con las distinciones señaladas entre los grupos comparados, de acuerdo a la categorización establecida por Baron con base en los promedios ponderados, se puede afirmar que en todas las subescalas se presenta una capacidad emocional "adecuada", dado que dichos puntajes se ubican en la categoría "promedio" según la clasificación que hace el autor.

El componente Interpersonal sigue la misma tendencia del componente Intrapersonal, es decir, son los voluntarios quienes presentan puntajes ponderados más altos frente a los no voluntarios, llegando a diferenciarse en 6 puntos en las subescalas "Relaciones interpersonales" y "Responsabilidad Social", no así en la subescala "empatía". No obstante estas diferencias y al considerar estos promedios ponderados (de acuerdo a la categorización de Baron), ambos grupos presentan un desarrollo "promedio" de esta capacidad emocional.

En el componente Adaptabilidad, si bien muestra diferencias menores entre ambos grupos, en la subescala "Solución de Problemas" el grupo de los voluntarios presenta un buen desarrollo de esa capacidad y resulta definida como "Capacidad Alta", a diferencia de los no voluntarios que permanecen en la categoría "Promedio". En las subescalas "Prueba de realidad" y "Flexibilidad", si bien los puntajes son mayores en los voluntarios, la clasificación teórica es la misma, correspondiendo a "promedio" en ambos casos.

El componente Manejo del Estrés refleja claramente, en sus promedios reales y ponderados, que los voluntarios superan a los no voluntarios, diferencia que se hace mayor en los puntajes ponderados (Baron, 2000), siendo de 4 a 8 puntos dicha diferencia. Además, es posible agregar que se observa una notable distinción en la subescala "Control de Impulsos", donde los voluntarios califican en "Capacidad Alta" y los no voluntarios en "Capacidad Promedio”.

Por último, en el componente Estado del ánimo en general, también los voluntarios superan en 4 a 8 puntos los puntajes ponderados y reales, a los no voluntarios. Sin embargo y aunque existe esta diferencia, al momento de contrastar con las categorías teóricas ambos grupos se ubican en "Capacidad Promedio", tanto en la subescala "Felicidad" como en "Optimismo". (Tabla N³). 
Tabla 3. "Promedios de subescalas por componente, Según condición de voluntario"3

\begin{tabular}{|c|c|c|c|c|c|c|}
\hline \multirow{2}{*}{$\begin{array}{l}\text { Subescalas Componente } \\
\text { Intrapersonal }\end{array}$} & \multicolumn{3}{|c|}{ Voluntarios $(\mathrm{N}=40)$} & \multicolumn{3}{|c|}{$\begin{array}{l}\text { No voluntarios } \\
(\mathrm{N}=262)\end{array}$} \\
\hline & & $\mathrm{Pp}^{3}$ & Ds & & $\mathrm{Pp}$ & Ds \\
\hline Conocimiento de sí mismo & 30 & 100 & 5.18 & 28 & 96 & 5.66 \\
\hline Asertividad & 26 & 98 & 4.6 & 26 & 98 & 4.8 \\
\hline Autoconcepto & 37 & 98 & 5.7 & 34 & 92 & 7.9 \\
\hline Autorrealización & 40 & 104 & 4.5 & 38 & 98 & 5.3 \\
\hline Independencia & 24 & 92 & 3.7 & 24 & 92 & 3.8 \\
\hline $\begin{array}{l}\text { Subescalas Componente } \\
\text { Interpersonal }\end{array}$ & & $\mathrm{Pp}$ & Ds & & $\mathrm{Pp}$ & Ds \\
\hline Empatía & 35 & 108 & 3.22 & 34 & 106 & 4.06 \\
\hline Relaciones Interpersonales & 47 & 104 & 4.39 & 44 & 98 & 6.42 \\
\hline Responsabilidad Social & 44 & 106 & 4.14 & 42 & 100 & 4.87 \\
\hline $\begin{array}{l}\text { Subescalas Componente } \\
\text { Adaptabilidad }\end{array}$ & & $\mathrm{Pp}$ & Ds & & $\mathrm{Pp}$ & Ds \\
\hline Solución de Problemas & 32 & 110 & 3.53 & 31 & 108 & 4.73 \\
\hline Prueba de Realidad & 38 & 96 & 5.57 & 37 & 94 & 6.35 \\
\hline Flexibilidad & 29 & 104 & 4.47 & 27 & 98 & 4.43 \\
\hline $\begin{array}{l}\text { Subescalas Componente } \\
\text { Manejo del estrés }\end{array}$ & & $\mathrm{Pp}$ & Ds & & $\mathrm{Pp}$ & Ds \\
\hline Tolerancia al Estrés & 33 & 98 & 5.19 & 31 & 94 & 6.05 \\
\hline Control de Impulsos & 39 & 116 & 6.4 & 36 & 108 & 5.9 \\
\hline $\begin{array}{l}\text { Subescalas Componente } \\
\text { Estado del ánimo en general }\end{array}$ & & $\mathrm{Pp}$ & Ds & & $\mathrm{Pp}$ & Ds \\
\hline Felicidad & 40 & 108 & 4.4 & 37 & 100 & 6.09 \\
\hline Optimismo & 33 & 100 & 4.44 & 31 & 96 & 5.31 \\
\hline
\end{tabular}

\section{Inteligencia Emocional y Variables demográficas y familiares en Voluntarios y No Voluntarios}

Al comparar los puntajes promedios que obtienen los jóvenes voluntarios y no voluntarios en la escala de inteligencia emocional con variables de índole familiar, muestra diferencias significativas sólo en la variable Ingreso familiar, y en los no voluntarios 
( $F=3.55 ; p<0.0151)$, donde aquellos que perciben ingresos sobre 810 mil pesos se diferencian significativamente en sus promedios de inteligencia emocional, respecto de quienes poseen ingresos de 400 mil y menos. Quienes tienen ingresos familiares entre 401 y 600 mil, no se diferencian de ninguno de los dos grupos mencionados. Si bien en el grupo de los voluntarios no se presentan diferencias significativas, los promedios mayores en inteligencia emocional los presentan aquellos que tienen entre 400 y 600 mil pesos de ingreso familiar (Tabla $\mathrm{N}^{\circ} 4$ ).

El mismo análisis con variables demográficas y académicas, muestra diferencias significativas respecto de la Religión ( $\mathrm{t}=-3.82$; $\mathrm{p}<.0002$ ) y de las Notas ( $\mathrm{t}=-2.06 ; \mathrm{p}<0.0403$ ) sólo en los No Voluntarios. Los promedios más altos los obtienen quienes no se identifican con alguna religión y que tienen promedios de notas más altos. La situación descrita también se presenta en los Voluntarios, sólo que en este grupo las diferencias observadas no resultan significativas.

Tabla 4. "Inteligencia emocional según Ingreso familiar y de Voluntarios y No Voluntarios"

\begin{tabular}{|c|c|c|c|c|}
\hline VARIABLES & $\begin{array}{l}\text { PROMEDIO } \\
\text { INTELIGENCIA } \\
\text { EMOCIONAL }\end{array}$ & $\mathrm{N}$ & \multicolumn{2}{|l|}{ Prueba } \\
\hline \multicolumn{5}{|c|}{ INGRESO FAMILIAR VOLUNTARIOS } \\
\hline Desde 810.000 y más & 108.6 & 11 & \multirow{4}{*}{$\mathrm{F}=0.72$} & \multirow{4}{*}{0.5478} \\
\hline Entre 610.000 y 800.000 & 103 & 4 & & \\
\hline Entre 400.001 y 600.000 & 111.5 & 17 & & \\
\hline Menos o igual a 400.000 & 103.7 & 7 & & \\
\hline TOTAL & & $39^{4}$ & & \\
\hline \multicolumn{5}{|c|}{ INGRESO FAMILIAR NO VOLUNTARIOS } \\
\hline Desde 810.000 y más & $102.7 \mathrm{~A}$ & 68 & \multirow{4}{*}{$F=3.55$} & \multirow{4}{*}{0.0151} \\
\hline Entre 601.000 y 800.000 & $102.2 \mathrm{~A}$ & 42 & & \\
\hline Entre 400.001 y 600.000 & $98.1 \mathrm{AB}$ & 73 & & \\
\hline Menos o igual a 400.000 & $92.9 \mathrm{~B}$ & 76 & & \\
\hline TOTAL & & $259^{5}$ & & \\
\hline
\end{tabular}

4.5 Se elimina categoría "No sabe" 


\section{Conclusiones}

La variable central del estudio es la Inteligencia Emocional, la cual fue estudiada y analizada en detalle -según la Escala de Baron (2000)-, cada una de las subescalas fue comparada según grupos de estudio: Voluntarios y No voluntarios.

Para los cinco componentes de Inteligencia Emocional Intrapersonal, Interpersonal, Adaptabilidad, Manejo del estrés y Estado del ánimo en general (Baron, 2000), incluida la tendencia de las subescalas, los puntajes resultantes -reales y ponderados- dan cuenta de que son los jóvenes voluntarios quienes presentan los mayores puntajes o bien puntajes similares, pero en ninguna ocasión los no voluntarios superan a su contraparte.

Ahora bien, al momento de clasificar los puntajes, utilizando las categorías del instrumento de medición de Baron (2000), ocurre que en la mayor parte de las subescalas, la clasificación es de Capacidad "Promedio" para ambos grupos, lo mismo ocurre a nivel de Componentes.

En lo específico, sólo en dos, de las quince subescalas, es posible encontrar distinciones explícitas - de acuerdo a esta categorización de Baron- entre voluntarios y no voluntarios. Esto es, en "Solución de Problemas" (perteneciente al componente Adaptabilidad) y en "Control de Impulsos" (componente Manejo del Estrés) los voluntarios presentan una Capacidad Alta, mientras que los no voluntarios muestran una "Capacidad promedio". Esto último es consistente con el estudio de Enciso y Lozano (2011), quienes logran concluir que el estilo cognitivo de los voluntarios se caracteriza por la reflexividad y que las estrategias cognitivas que utilizan, generalmente para la solución de problemas sociales, guardan relación con la habilidad en la observación y retención de información relevante sobre la situación, así también por la búsqueda de soluciones alternativas, la anticipación de las consecuencias que pueden tener sus comportamientos y la elección de los medios adecuados según los fines que se persiguen.

Al momento de comparar el nivel de Inteligencia emocional global, de acuerdo a las categorías establecidas por Baron, los voluntarios se concentran mayoritariamente en las categorías "alta" y "promedio" (35\% en cada una), mientras que los no volunta- 
rios presentan la categoría "promedio" con mayoría porcentual (49,6\%); es más, si sólo se consideran las categorías "Alta” a "marcadamente alta", son los voluntarios los que concentran allí el $52,5 \%$ de ellos, versus el $26 \%$ de los no voluntarios, diferencias que resultan significativas entre voluntarios y no voluntarios en el puntaje de Inteligencia Emocional global ( $t=-2.97, p<0.0032)$, confirmando con ello uno de los supuestos de esta investigación: Los jóvenes que realizan actividades de voluntariado tienen un mayor desarrollo de su inteligencia emocional, frente a aquellos que no realizan estas actividades; consistente además con lo que expone Zulueta (2003) respecto al doble aporte del voluntariado; por un lado, ayudar a quienes lo necesitan (siendo el más convencional) y por otro, fortaleciendo en quienes lo ejecutan, valores como la solidaridad, la responsabilidad social y empatía, los cuales estarían vinculados al concepto de inteligencia Emocional. En conjunto, esto es lo que la ONU (2000) plantea como el "segundo beneficio" del voluntariado, es decir que además de un beneficio económico (tras el ahorro que se produce al no ser un trabajo remunerado) aporta a la sociedad en su conjunto, ya que por medio de la potenciación de estas habilidades y/o valores, se retribuye a la sociedad ciudadanos más "completos" y que pueden aportar con mayores herramientas en la cultura en que se insertan.

Al profundizar el análisis de la Inteligencia Emocional y comparar en virtud de otras variables personales y académicas, diferenciadas por grupo de Voluntarios y no voluntarios, es posible afirmar que se encontraron diferencias significativas de acuerdo a la religión y promedio de notas, sólo en los no voluntarios; presentan promedios de inteligencia emocional mayor quienes no se identifican con una religión y tienen notas más altas, tendencia que es similar en los voluntarios, pero allí las diferencias no son significativas. El análisis de variables familiares arrojó diferencias significativas con el ingreso familiar en los no voluntarios, siendo los que tienen un mayor ingreso (sobre 810 mil pesos) quienes presentan también mayores promedios de inteligencia emocional, lo cual difiere de la realidad de los voluntarios, ya que en este grupo el puntaje mayor lo obtienen quienes se encuentran entre 400 y 600 mil pesos.

Se concluye así que los voluntarios suelen tener un nivel de inteligencia emocional mayor, observando también que aquellos que 
tienen mejores notas y un ingreso medio también presentan esta característica, lo que sugiere ideas para próximas investigaciones que vinculen predictores exactos de la Inteligencia Emocional. Por último, si se sigue esta lógica de que aquellos que realizan acciones de voluntariado tienen un nivel de inteligencia emocional mayor, lo que se traduce en seres humanos capaces de comprenderse a sí mismos, asertivos, que mantienen buenas relaciones con otros, capaces de resolver problemas, entre otras características; no cabe duda que el potenciar actividades de voluntariado en cualquier país, es una política pública beneficiosa, ya que no sólo se está generando una red de acción poderosa, sino que a su vez se está mejorando la calidad humana de la sociedad. Es así como el Trabajo Social toma protagonismo, ya que se vincula con esta realidad tanto en los espacios del quehacer directo con organizaciones de voluntarios, como en el diseño de programas y políticas, por lo que encuentra una oportunidad de promover y potenciar estas acciones a la luz de estos y otros hallazgos que dan cuenta del efecto múltiple y positivo de participar de acciones de voluntariado.

\section{Bibliografía}

Alcalá Consultores (2002). Voluntariados en Chile: Lo plural y lo diverso. Sistematización de antecedentes generales en Chile y el exterior. Santiago de Chile: División de Organizaciones Sociales del Ministerio Secretaría General de Gobierno.

Bar-On, R. (2000). Emotional and social intelligence: insights from the Emotional Quotient Inventory (EQ-i). In R. Bar-On E J.D.A. Parker (eds.): Handbook of emotional intelligence. San Francisco: Jossey-Bass.

Delgadillo, L. (2005). El voluntariado universitario y su importancia para las universidades. En II Reunión de la Red Latinoamericana de Voluntarios Universitarios en apoyo a UNITES. Pernambuco, Recife, Brasil. Recuperado de http://unites.ucol.mx/pdf/2da_ reunion/voluntariado_ligia_delgadillo.pdf

Enciso, E. E Lozano, M. (2011). Diferencias en actitudes y estrategias cognitivas sociales en jóvenes vinculados y no vinculados a programas de voluntariado. Psychologia. Avances de la disciplina, 5 (2) pp. 81-94. Recuperado de http://www.redalyc.org/articulo. oa?id=297224105007 
Goleman, D. (1995). Inteligencia Emocional. Buenos Aires, Argentina: Javier Vergara Editores.

Goleman, D. (2006). Inteligencia Social, México: Planeta Mexicana, S.A. Organización Mundial de las Naciones Unidas (2000). Voluntariado y Desarrollo Social. Documento de antecedentes para discusión en la reunión del grupo de expertos. Nueva York, 29 y 30 de noviembre de 1999. 2000. Recuperado el 1 abril, 2013. De http://www.boluntariotza.net/comun/biblioteca/ voluntariado_y_desarrollo_social_ONU.pdf

Rodríguez, E. (2000). Voluntariado, participación, equidad y ciudadanía: Los jóvenes como actores estratégicos del desarrollo. Notas presentadas en Prácticas óptimas en torno al voluntariado juvenil, organizado por la Dirección Nacional de la Juventud, Buenos Aires, Argentina.

Román, J.; Ibarra, S. (2011). ¿Cómo es el voluntariado en Chile? Revista Psicología hoy, Vol N9. Facultad de Psicología, Universidad Alberto Hurtado. Recuperado de http://psicologia.uahurtado.cl/wp-content/ uploads/2011/10/psh9-web-ok-1.pdf

Salovey, P.; Mayer, JD. (1990). Inteligencia Emocional. Imaginación, Conocimiento y Personalidad, 9 (3), pp. $185-211$.

Sotil, A. y Quintana. A. (2002). Influencias del clima familiar, estrategias de aprendizaje e inteligencia emocional en el rendimiento académico. Revista de Investigación en Psicología, Vol.5, No.1, Julio 2002. Recuperado el 8 de agosto, 2012. De http:// sisbib.unmsm.edu.pe/bvrevistas/investigacion_ psicologia/v05_n1/pdf/a03v5n1.pdf

Ugarriza, N. (2001). Evaluación de la Inteligencia Emocional a través del inventario de BarOn (I-CE) en la Muestra de Lima Metropolitana. Persona, $N^{\circ} 4$, pp. 129-160. Perú: Universidad de Lima.

Zulueta, S. (2003). La Evolución del Voluntariado en Chile entre los años 1990 y 2002. Tesis para postular al grado de Magíster en Sociología. Marzo, 2003, Universidad Católica de Chile, Santiago, Chile. 
